\title{
The Relationship Between Parental Care and Pain in Children With Headache: A Narrative Review
}

\author{
Giulia Natalucci; Noemi Faedda, MD, PhD; Valentina Baglioni, MD, PhD ; Vincenzo Guidetti, MD
}

Purpose.-In migraine or primary headache in children, parents play a fundamental role in pain management. For this narrative review, PubMed, Google Scholar, and Psych Info were searched using the terms "parent headache", "mother/ father headache", "parental impact headache", "alexithymia parents headache", "catastrophizing parent headache", "family headache", "children parent headache", and "quality of life family headache". Articles were chosen for inclusion based on their relevance in to the topic.

Overview.-Several parental and psychological characteristics can influence in children and adolescent headache, such as parental attitudes as oppressive or overprotective; punitive parenting styles; familial psychological symptoms, especially anxiety and depression; catastrophizing about their child's pain or excessive worry about their child's headache; inability to express emotions; and feelings that may lead to somatization problems.

Discussion.-Parents' attitudes and behaviors toward their child's headache have a strong relation with the severity of headache attacks. Mothers seem to have more influence than fathers on children's pain and emotional regulation. We suggest that the presence of caregiver-child transmission of maladaptive coping strategies, arising from difficulties expressing emotion, may lead to incorrect management of headache pain, further facilitating headache chronification.

Key words: parents, headache, pain, children, adolescents, care

\section{INTRODUCTION}

Headache in childhood has a multifactorial etiology. Many studies have not only noted a strong genetic predisposition ${ }^{1}$ but also the involvement of environmental factors, ${ }^{2}$ such as exposure to stressful events, dysfunctional family situations, physical or emotional abuse, ${ }^{3}$ bullying by peers, ${ }^{4}$ or lifestyle factors, such as obesity and low level of physical activity. ${ }^{5,6}$

Not rarely, headache is comorbid with other neurological and psychiatric disorders. Among the psychiatric disorders, internalizing disorders are frequently reported, such as anxiety, especially separation, generalized and school anxiety, and depression. ${ }^{7-9}$ Twin studies have demonstrated common genetic

From the Department of Human Neuroscience, Sapienza University of Rome, Rome, Italy (G. Natalucci, N. Faedda, V. Baglioni, and V. Guidetti).

Address all correspondence to V. Guidetti, Department of Human Neuroscience, Sapienza University of Rome, Via dei Sabelli 108, 00185, Roma, Italy, email: vincenzo.guidetti@ uniroma1.it

Accepted for publication April 6, 2020.

Headache

(C) 2020 American Headache Society features underling the comorbidity of anxiety and depression with migraine, ${ }^{10,11}$ and neurobiological studies have suggested altered serotonin and dopamine pathways as the putative cause of the frequent comorbidity of internalizing disorders and migraine. ${ }^{12-14}$

Among the environmental factors that can affect the onset and maintenance of headaches in children and adolescents, the role of parents' behavior seems to be relevant, although it has not yet been deeply studied. ${ }^{15}$ In fact, elements such as emotional regulation, attachment, and educational styles, and the presence of dysfunctional coping styles and psychopathologies in mothers, can negatively affect headache in children. Specifically, parental attitudes, such as maintaining a high level of control over the child or frequently suppressing their independence, are known to be factors associated with increased migraine attack frequency and severity in children and adolescents. ${ }^{16}$ Moreover, how parents respond to children's pain plays a central role in conceptual models of the development and maintenance of pain-related behaviors in children. ${ }^{17}$ Children and adolescents learn to modulate their responses and behaviors based on parental feedback. If responses are inappropriate, the child may exhibit dysfunctional behaviors, and in the perspective of headaches, the frequency and severity of attacks can increase, potentially facilitating further chronification. ${ }^{18}$

One theory is that learned pain responses could be due to intergenerational transmission, in which parents learn to manage pain from their respective parents, and then transmit these coping strategies to their children. Hatchette et al. demonstrated this hypothesis using qualitative interviews with dyads of mothers and adolescents. ${ }^{19}$ They found a clear intergenerational transmission of information about pain and pain management.

Because pain in children is often a disabling condition, and involves not only the person with headache but also their family and social environment, it is important to analyze the impact that parents can have on the pain of children, and how they can influence migraine and headache in their children.

In this article, we will emphasize the role that parents play in the management of headache-related pain in their children. We will analyze how pain in children affects the family system, and how having a parent who also suffers from headache can further affect the functioning of both the individual and the family. Finally, we will analyze some factors related to parents' attitudes, such as attachment style, emotional regulation, and catastrophic thoughts.

Conflict of Interest: None 


\section{THE ROLE OF PARENTS ON CHILDREN HEADACHE}

The management of a young person with chronic pain in general, and headache in particular, can be demanding for parents. Chronic pain can negatively affect both the child and family, leading to several social restrictions, higher stress levels, symptoms of depression and anxiety, and a sense of inadequacy and helplessness. ${ }^{20}$ It can also affect normal family functioning through changes in everyday life (eg, frequent medical examinations, work permits to take care of their child, reduction of sports or recreational activities, etc.). ${ }^{21}$

Development of headaches in children and adolescents can become a relevant stressor for parents, and this high stress level may influence, in turn, the child's experience of headache, affecting their ability to regulate emotions and behavior. Operto et al compared an adolescents group with primary headache diagnosis to a group of healthy adolescents. In this study results revealed no statistically significant difference in reported parenting stress level between the groups overall. However, analysis of the data did reveal that the frequency of headache attacks was associated with increasing parental stress, suggesting that headache frequency rather than disease duration can increase the risk of parental stress. ${ }^{22}$

Parenting stress is a relevant matter, especially in headache and chronic pain, because it can influence the management of the pain condition and lead to dysfunctional child behaviors. This is probably due to a transactional relationship between the child's behavioral problems and parenting stress. ${ }^{23}$ It can be assumed that a circular relationship exists among cephalalgic pain in the child, parental stress, and dysfunctional behaviors in the child, with a possible tendency toward headache chronification. It is, therefore, important to always evaluate the stress level within the family unit and identify the dysfunctional adopted solutions, in order to best assist in the management of stressful situations due to headache or migraine. ${ }^{24}$

In addition to the negative effect that parental stress can have on childhood headache, some studies have identified several parent and family factors that seem to influence the pain experience of the children, such as pain beliefs, worries, catastrophic thoughts, illness behaviors, as well as parental protective, minimizing, encouraging, or monitoring responses. ${ }^{25,26}$

In regards to parents and headache, Wall and colleagues utilized the Parent Response Scale, which assesses both child and parent perceptions of parental responses to headache episodes, distinguishing the responses from children and adolescents. They found that higher levels of parent-perceived negative responses were associated with higher levels of reported behavioral problems in adolescents, whereas higher levels of parent-perceived affective responses were associated with lower levels of parent-reported functional disability in younger children. ${ }^{18}$ This may suggest that a generalized punitive parenting style can result in more general behavioral problems for adolescents, whereas a more supportive and empathic response to headache-related behavior may reduce adolescents-associated behavioral problems. On the other hand, for children younger than 11 years, re-enforcement with positive feedback during symptom-free intervals, using supportive comments, presenting entertainment/distractions during pain, or selectively ignoring dysfunctional pain-related behaviors, appears to be the best strategy for parents. These findings support the idea that training parents on more effective and educational responses to their child's headache pain may assist in avoiding problematic behaviors.

Another study compared children with migraine and their parents to a control group. This study analyzed the presence of psychiatric comorbidities (anxiety, depression, and eating disorders) in subjects with migraine in relation to parental attitudes, using the Parenting Style Inventory. Results revealed that parents of children with migraine granted significantly less autonomy to their children compared to parents from the control group. Moreover, parents of children with chronic headache appeared to be much more sensitive about matters concerning their children's health, exhibiting overprotective attitudes. ${ }^{27}$ This finding is partially confirmed by a previous study, in which parents of children with migraine displayed a more protective attitude compared to parents of children with $\mathrm{TTH}^{28}$

Finally, the psychological symptoms were more frequent in the migraine group than in the control group and, interestingly, mothers from the migraine group had more somatic symptoms than mothers from the control group. This may confirm that pain and somatization can be transmitted from mother to child.

Arruda and colleagues demonstrated the transmission finding that headache status in the mother is associated with headache status in the children. ${ }^{29}$ In fact, in a sample of 1994 children (5-12 years), it was shown that when the mother had a low frequency of headaches, the child was also more likely to have a low or intermediate headache frequency. Moreover, when the mother had chronic daily headache $(\mathrm{CDH})$, risk of $\mathrm{CDH}$ in their children was increased almost 13-fold, and if mothers had high frequency forms of headache, children were more likely to have high frequency forms as well.

Another important aspect, also reported by numerous studies, is the impact of headaches on children's school performance. ${ }^{30}$ In the light of the apparent relationship between adolescent headache and parental attitude, Kaczynski et al analyzed the impact of parenting behaviors on school functioning in adolescents with migraine and tension-type headache (TTH). This study revealed a relationship between 
parental protective behavior and difficulties with school functioning in children, especially for those with TTH compared to chronic migraine (CM). ${ }^{31}$ To explain this data, the authors indicated the likely relevant role of the higher frequency and longer duration of TTH, compared to migraine. Because of the characteristics of TTH, adolescents reported greater difficulties with school functioning in general (missed class work or too many schooldays lost). Additionally, because school is an important trigger for headache attacks, parents may present more protective and apprehensive behaviors in order to prevent or limit the recurrent episodes. This parental attitude may actually increase the level of dysfunction with further exacerbation of TTHs, due to increased parental protectiveness that perpetuates the vicious cycle of pain and disability. The impact of pain and its consequences on child functioning can lead to an excessive protective behavior in parents, allowing their children to avoid school and to receive extra attention, potentiating further school-related problems. Although parents believe they are acting in the child's best interest in helping to reduce the pain, these behaviors are actually deleterious for normal school functioning and performance.

Most of the research examining the relationship between children and adolescents with headache and their respective parental attitudes toward the headache conditions were conducted in specialist headache centers. In contrast, Samsmaz et al. enrolled parents of children with headache diagnosis in a school, wondering how aware parents were of their children's headaches. ${ }^{32}$ Parents of children with headache did appear to be aware of their child's headache episodes. The most relevant factors that influenced parental awareness were objective and specific symptoms, such as severity of the migraine attacks, nausea, vomiting, and light sensitivity. Further factors playing an important role were female gender, being the first child of the family, history of motion sickness, and family history of headaches. Interestingly, parents without a history of headache were less aware of their children's headaches, suggesting an underestimation of the problem.

Similarly, Nakamura et al. enrolled a representative sample of adolescents in the United States (6483 adolescents aged 13-18 years), with the aim of assessing the concordance between parent and adolescent headache ratings, and if it was influenced by parental and adolescent gender, age of the child, or type of headache. ${ }^{33}$ In contrast to Samsamaz, they found a relevant discrepancy between the headache frequency reported by parents. In fact, the frequency reported by adolescents was much higher than those reported by parents. Focusing on the difference between parents, they found that mothers/female caregivers were more likely to recognize headaches in their offspring than fathers/male caretakers, and female caregivers were more likely to report headaches in their male offspring.
It was postulated that female caregivers were more accurate in reporting headaches because they spent more time with their children, and because adolescents were more likely to reveal information to and ask their mother's for care than their fathers. ${ }^{34,35}$

In the literature there are very few studies that have involved both parents in assessing their child's headache. In a study conducted by Ertem et al both parents were included in assessing headache characteristics, depression, anxiety, and parental attitudes in the pediatric population with episodic migraine (EM), CM, and TTH. ${ }^{15}$ In regard to psychological problems, pain intensity had a statistically significant positive correlation in all three groups. Interestingly, father and mother attitudes were different. The father's attitude was not significantly correlated with the development of psychiatric symptoms and headache severity, whereas a mother's attitude toward children with EM was related to higher anxiety symptoms. Moreover, in children with CM, mothers had a significantly higher oppressive-authoritarian attitude than in the other headache groups, resulting in a relevant impact on the child's pain experience.

Børte and colleagues confirmed the hypothesis that mothers have more influence on their children's pain perception than fathers. In fact, they found a strong relationship between parental migraine and migraine in their offspring. ${ }^{36}$ Specifically, both maternal and paternal migraine were significantly associated with migraine in their offspring, but mother-offspring associations were significantly stronger than father-offspring associations. These data suggest that mothers have a stronger influence on their children's pain behavior than fathers, as they are more often the primary caregivers.

\section{IMPACT OF PARENTAL HEADACHES ON THEIR CHILDREN}

We have seen how parental behaviors or attitudes can affect their children's headaches in terms of severity or frequency. Now, we will focus on the role that parental headache has on their children, both with or without headaches. This is an important matte because chronic pain in a parent can have a significant impact on the family and child functioning. In fact, it has been demonstrated that chronic pain in mothers may lead to a reduction in their parental duties, and this may lay the foundations for behavioral problems in children. ${ }^{37}$

Through a validated, computer-assisted, telephone interview, it was demonstrated that people with migraine have more problems communicating with their partner or child, argue more frequently, felt less involved with home and school activities, and spent less time with their children. ${ }^{38}$ In contrast with men, most women with migraine felt they could be better parents if 
they did not have migraine. These negative feelings referred to perception of an inadequate parental role due to migraine pain, which can have relevant consequences on children's behaviors. For example, the child could be at risk of assuming inappropriate or premature responsibilities before they are sufficiently autonomous for this domain, such as replacing the parent in his/ her educational role. ${ }^{39}$

As is well known, migraine has a negative impact on quality of life. ${ }^{40} \mathrm{~A}$ comparison between mothers with and without migraine demonstrated that mothers with migraine headaches report impaired quality of life and more symptoms of depression and anxiety than a control group. ${ }^{41}$ Moreover, maternal characteristics, such as anxiety and depressive symptoms (and, in general, her emotional status), age, and severity of migraine headaches, can negatively affect their children's quality of life, especially regarding social and school relations.

Having a parent with migraine may be deleterious for several child functioning areas. In the CaMEO study, the authors used data collected directly from children who had a parent with migraine. In all examined domains (Loss of Parental Support and Reverse Caregiving, Emotional Experience, Interference with School, Missed Activities, and Events), the perceived burden of parental migraine was greater for those who had a parent with CM than EM. ${ }^{42}$ An interesting result concern was that adolescents reported that they were not receiving help from their parents when requested due to their migraine condition. These data were confirmed by another study in which the impact on children's global well-being (physical and emotional health, sleep, impact on school), relationship with other family members, and lack of help (eg, taking care of the parent) are negatively impacted by the severity and frequency of migraine attacks in their parents. ${ }^{43}$

Contrary to these findings, one study reported that when children and mothers with migraine were compared to children without migraine, the results changed. ${ }^{44}$ In a large population-based study, headache status or frequency in the mother did not appear to influence behavior in children with migraine, but had a stronger influence on children without headache. Child Behavior Checklist (CBCL) scores demonstrated that headache frequency in the mother was positively associated with internalizing symptoms in children without headaches. Interestingly, when the mother had daily headaches, the CBCL scores were not very different between the two groups. So, it seems that children without migraine may be more affected by maternal headache frequency than children with headache. This is an interesting point for reflection: When living with a mother with headache, do children with headache learn more adaptive coping strategies than children without headache? Do they learn to regulate their emotions and behaviors based on this problem?
The last aspect to be explored is the use of drugs in children with headaches, and how parenting behaviors and psychological symptoms can have a negative impact on this. It seems that the severity and the burden of parental headache are associated with their children's use of medicine for headache. Moreover, gender differences might be relevant, as the use of medicine for headache in girls is influenced by both mother's and father's headache and by the severity of their headache symptoms, whereas in boys drug use was only associated with the mothers' headache and the severity of their headache symptoms. ${ }^{45}$

\section{HEADACHE AND ALEXITHYMIA, EMOTIONAL REGULATION, AND ERRONEOUS THINKING IN PARENTS}

In this section, we will focus on psychological issues related to emotional regulation in both parents and children, and how these can influence headache management and pain.

As previously analyzed, parental attitudes play a central role in the exacerbation and maintenance of pediatric headache, ${ }^{46}$ but what is the role of emotion in this process?

In normal conditions, children learn to regulate their internal and external states, arousal, and behavior during infancy through interactions with their caregivers. Gradually, children learn how manage with their own actions and emotions, practice self-regulating behaviors, and how to apply this information in every-day life experiences. ${ }^{47,48}$ So, if parents are not able to regulate their emotions and feelings, or to express them in the right way in the right context, there may be consequences to their children's ability to self-regulate emotional states. Therefore, the predisposition for somatization and internalizing problems may be more prevalent.

This emotional inability is defined as alexithymia, which is a psychological factor that has received considerable attention in the last decades. Alexithymia describes one's difficulty in distinguishing and describing various emotions, having a restricted imagination, and manifesting a tendency toward externally oriented thinking rather than internally oriented thinking. ${ }^{49}$ Mothers with alexithymia have a limited capacity to experience positive emotions, such as joy and happiness in their relationships, and they are not empathetic and effective in modulating the emotional states of their children. ${ }^{50}$

Mothers with alexithymia and depressive symptoms can have a direct and negative influence on the quality of their interactions with their children, ${ }^{51}$ and children with migraine present higher alexithymic levels than healthy peers. 52,53 Moreover, in a sample of adolescents and mothers both suffering from migraine, the teens had higher levels of alexithymia compared to migraine-free adolescent and mother controls. ${ }^{54}$ 
This assumes a possible intergenerational transmission of alexithymia from mother to child, with a mother's deficit in expression of feelings and emotional regulation negatively affecting her children.

Another study assessed the role of maternal attachment style and alexithymia on childhood headache severity (intensity and frequency) and psychological profile (anxiety, depression, and somatization). This study showed that maternal alexithymia levels and attachment style did not appear to influence childhood migraine severity. ${ }^{55}$ Moreover, alexithymia traits appeared to influence their children's attachment style and affective regulation.

A relationship between attachment style and alexithymia has been indicated in some studies on alexithymia. ${ }^{56}$ More specifically, individuals who have an insecure attachment style appear to have higher alexithymia levels compared to securely attached subjects. $^{57}$

Finally, the relationship between maternal alexithymia levels and separation anxiety, anxiety related to school, and children's feeling of guilt seems very strong. This confirms the interrelation among internalizing symptoms, somatization, and difficulties in expressing emotions.

In addition to these psychological problems, alexithymia is frequently associated with perceptual or attentional bias, and catastrophic thoughts on pain.

The study on headache and alexithymia by Shim et al. aimed to understand how alexithymia influenced the quality of life in individuals with headache, and the impact of other psychological characteristics, such as anxiety and depression symptoms, somatization, and pain catastrophizing. ${ }^{58}$ Among these variables, they found that pain catastrophizing was the only significant pathway through which alexithymia was related to headache impact, even after considering the impact of anxiety and depression. This result suggests that alexithymia may be a more distal risk factor, affecting headache impact through its association with somatization or pain catastrophizing. Therefore, the association between emotional and affective dysfunctions, with erroneous thinking or cognitive bias, may exacerbate the headache impact on an individual through internal and emotional states.

The definition of pain catastrophizing is an exaggerated negative orientation to actual or anticipated pain. ${ }^{59}$ Catastrophizing thoughts about pain are defined as an exaggerated, and negative orientation against actual or expected experiences of pain, which include some cognitive distortions, such as rumination (tendency to focus on pain thoughts), magnification (tendency to exaggerate the threat expression related to the pain), and helplessness (tendency to present a helpless attitude in coping with the experience of pain). ${ }^{60}$
In childhood migraine, catastrophizing thoughts and fear of pain are not uncommon. ${ }^{61}$ It seems that this attitude, together with internalizing symptoms, can cause a general decline in the quality of life, with a probable parents' underestimation of the problem. ${ }^{62}$ This tendency to catastrophize on pain is not related exclusively to $\mathrm{CM}$; indeed, it could lead to the chronification of headaches in general.

What happens if parents experience catastrophic thoughts about their child's pain? In general, these thoughts in parents have been linked to an increase in medication use targeted toward pain control. ${ }^{63}$ Negative parental attitudes toward pain can also increase childhood disability, by influencing pain severity and the child's ability to function. ${ }^{64}$ This can be explained by the pain circle, in which a parent's dysfunctional strategy toward their child's pain may lead to an inability for the child to develop adaptive strategies for pain management, increasing their overall pain severity, and further promoting the maladaptive process. Akbarzadeh et al investigated how parental catastrophic thinking can influence the emotional interpretation of their child's headache experiences. They included children with chronic headache, and found that mothers had a higher level of pain catastrophizing than fathers. ${ }^{65}$ Mothers with higher levels of pain catastrophizing reported more severe pain intensity in their children, whereas father's catastrophizing had no contribution to the reports of pain intensity in their children. The tendency for mothers to have greater catastrophizing thoughts than fathers was also reported by another study which demonstrated that parents' catastrophizing is associated with greater attempts to control both pain and everyday life activities in their children. ${ }^{66}$ This evidence is important because in many pediatric clinics mothers play a large role in reporting and clarifying their children's reports of pain, and their bias can in part influence the recommended medical and pharmacological interventions.

A positive correlation between alexithymia and pain catastrophizing has also been observed for other pain disorders, such as fibromyalgia, ${ }^{67}$ and in patients with chronic pain, ${ }^{68}$ suggesting a common pathway among different chronic forms of pain, impairments of emotional expression, and cognitive distortions.

\section{DISCUSSION}

Although we cannot determine whether the higher level of psychological factors seen in headache subjects represents vulnerability factors as part of the headache condition itself, or are a consequence of the headache experience, it is evident that these psychological factors must be addressed in the management of headache patients. Similarly, primary headache disorders 
in childhood represent not only a neurological condition but also present with concomitant psychological and familial components. Parents, especially mothers, play a relevant role in the management and childhood experience of pain, through the learned behavior of adaptive coping strategies, emotional regulation, and reaction to their child's headache and pain.

During childhood, the parents' ability to be aware of their child's problems is the most important factor. For this reason, children need close attention and encouragement from their parents in any situation, especially in medical conditions. Lack of awareness may cause a delay in the diagnosis and treatment of headache in childhood, and may additionally have an adverse effect on their education and social lives. On the other hand, an excessive attention to their child's pain conditions may lead to an overestimation of the problem, and promote further psychological consequences (eg, anxiety, depression, problems at school and with peers, and chronification of pain).

There are some limitations in the published research evaluating the role of parents on their children's headaches. As explained, mothers are the caregivers who most often accompany their children during medical visits. In these studies, researchers often used a parent self-report questionnaire to assess the pain experienced by the children, which was most often compiled by mothers. ${ }^{69}$ This could be a relevant methodological bias influencing the pain impact results on children due to the potential overestimation of pain reported by mothers. Moreover, both mothers' and fathers' underlying psychological symptoms or cognitive bias (eg, catastrophic thinking, idealization, and high or low expectations for children) could interfere with their ability to accurately answer questionnaires regarding symptoms experienced by their children. ${ }^{70}$

\section{CONCLUSION}

In conclusion, it is important to involve the family unit and consider the child's environmental experiences when formulating a plan for the treatment of childhood headache. Treatment should include both pharmacological and psychological management strategies when appropriate. Parents may need training in how to adequately manage their child's headache pain, and cognitive behavioral therapies should be considered for both the child and the family unit in order to reduce stress levels, and manage anxiety and depressive symptoms.

\section{KEY CONCLUSIONS}

- There appears to be a circular relationship among parental stress, dysfunctional behaviors in the child, and the development of further headache chronification.
- It is important to evaluate the stress level within the family unit and identify any dysfunctional adopted solutions in order to best assist in the management of stressful situations due to headache or migraine

- Higher rates of psychological and somatic comorbidities are seen in children with migraine who have parents who also experience psychological and somatic comorbidities, or are overprotective

- Children of mothers with high headache frequency are more likely to experience high headache frequency themselves

- A mother's behavior and attitude has a greater influence on the childhood experience and perception of pain than that of the father

- Alexithymia, the inability to identify or express one's emotions, appears more common in children whose parents struggle with their own ability to express and regulate their emotions. This increases the predisposition for pediatric somatization and internalizing disorders

- Parents, especially mothers, play a relevant role in the management and childhood experience of pain, through the learned behavior of adaptive coping strategies, emotional regulation, and reaction to their child's headache and pain

- Mothers have a higher tendency for pain catastrophizing than fathers, and estimate more severe pain intensity is experienced by their children

\section{References}

1. Gormley P, Anttila V, Winsvold BS, et al. Meta-analysis of 375,000 individuals identifies 38 susceptibility loci for migraine. Nat Genet. 2016;48:856-866.

2. Kröner-Herwig B, Gassmann J. Headache disorders in children and adolescents: Their association with psychological, behavioral, and socio-environmental factors. Headache. 2012;52:1387-1401.

3. Tietjen GE. Childhood maltreatment and headache disorders. Curr Pain Headache Rep. 2016;20:26.

4. Gini G, Pozzoli T, Lenzi M, Vieno A. Bullying victimization at school and headache: A meta-analysis of observational studies. Headache. 2014;54:976-986.

5. Bigal ME, Lipton RB. Obesity is a risk factor for transformed migraine but not chronic tension-type headache. Neurology. 2006;67:252-257.

6. Russo A, Bruno A, Trojsi F, Tessitore A, Tedeschi G. Lifestyle factors and migraine in childhood. Curr Pain Headache Rep. 2016;20:9.

7. Antonaci F, Nappi G, Galli F, Manzoni GC, Calabresi P, Costa A. Migraine and psychiatric comorbidity: A review of clinical findings. J Headache Pain. 2011;12:115-125.

8. Anttila P, Sourander A, Metsähonkala L, Aromaa M, Helenius H, Sillanpää M. Psychiatric symptoms in children with primary headache. J Am Acad Child Adolesc Psychiatry. 2004;43:412-419.

9. Dindo LN, Recober A, Haddad R, Calarge CA. Comorbidity of migraine, major depressive disorder, and generalized anxiety disorder in adolescents and young adults. Int J Behav Med. 2017;24: 528-534. 
10. Schur EA, Noonan C, Buchwald D, Goldberg J, Afari N. A twin study of depression and migraine: Evidence for a shared genetic vulnerability. Headache. 2009;49:1493-1502.

11. Ligthart L, Nyholt DR, Penninx BW, Boomsma DI. The shared genetics of migraine and anxious depression. Headache. 2010;50:1549-1560.

12. Breslau N, Lipton RB, Stewart WF, Schultz LR, Welch KM. Comorbidity of migraine and depression: Investigating potential etiology and prognosis. Neurology. 2003;60:1308-1312.

13. Noseda R, Kainz V, Borsook D, Burstein R. Neurochemical pathways that converge on thalamic trigeminovascular neurons: Potential substrate for modulation of migraine by sleep, food intake, stress and anxiety. PLoS ONE. 2014;9:e103929.

14. Barbanti P, Fabbrini G, Ricci A, et al. Migraine patients show an increased density of dopamine D3 and D4 receptors on lymphocytes. Cephalalgia. 2000;20:15-19.

15. Ertem DH, Bingol A, Ugurcan B, et al. The impact of parental attitudes toward children with primary headaches. Clin Child Psychol Psychiatry. 2019;24:767-775.

16. Siniatchkin M, Gerber WD. Role of family in development of neurophysiological manifestations in children with migraine. Prax Kinderpsychol Kinderpsychiatr. 2002;51:194-208.

17. McGrath PA, Seifert CE, Speechley KN, Booth JC, Stitt L, Gibson MC. A new analogue scale for assessing children's pain: An initial validation study. Pain. 1996;64:435-443.

18. Wall BA, Holden EW, Galdstein J. Parent responses to pediatric headache. Headache. 1997;37:65-70.

19. Hatchette JE, McGrath PJ, Murray M, Finley GA. Maternal influences in adolescents' pain self-management: A qualitative investigation. Vulnerable Child Youth Stud. 2006;1:159-169.

20. Eccleston C, Crombez G, Scotford A, Clinch J, Connell H. Adolescent chronic pain: Patterns and predictors of emotional distress in adolescents with chronic pain and their parents. Pain. 2004;108:221-229.

21. Wallace DP, McCracken LM, Weiss KE, Harbeck-Weber C. The role of parent psychological flexibility in relation to adolescent chronic pain: Further instrument development. J Pain. 2015;16:235-246.

22. Operto FF, Craig F, Peschechera A, Mazza R, Lecce PA, Margari L. Parenting stress and emotional/behavioral problems in adolescents with primary headache. Front Neurol. 2018;8:749.

23. Neece CL, Green SA, Baker BL. Parenting stress and child behavior problems: A transactional relationship across time. Am J Intellect Dev Disabil. 2012;117:48-66.

24. Esposito M, Gallai B, Parisi L, et al. Maternal stress and childhood migraine: A new perspective on management. Neuropsychiatr Dis Treat. 2013;9:351-355.

25. Jordan A, Eccleston C, Crombez G. Parental functioning in the context of adolescent chronic pain: A review of previously used measures. J Pediatr Psychol. 2008;33:640-659.

26. Palermo TM, Chambers CT. Parent and family factors in pediatric chronic pain and disability: An integrative approach. Pain. 2005;119:1-4.

27. Kandemir G, Hesapcioglu ST, Kurt ANC. What are the psychosocial factors associated with migraine in the child? Comorbid psychiatric disorders, family functioning, parenting style, or mom's psychiatric symptoms? J Child Neurol. 2018;33:174-181.
28. Claar RL, Simons LE, Logan DE. Parental response to children's pain: The moderating impact of children's emotional distress on symptoms and disability. Pain. 2008;15:172-179.

29. Arruda MA, Guidetti V, Galli F, Albuquerque RC, Bigal ME. Frequency of headaches in children is influenced by headache status in the mother. Headache. 2010;50:973-980.

30. Rousseau-Salvador C, Amouroux R, Annequin D, Salvador A, Tourniaire B, Rusinek S. Anxiety, depression and school absenteeism in youth with chronic or episodic headache. Pain Res Manag. 2014;19:235-240.

31. Kaczynski KJ, Claar RL, Lebel AA. Relations between pain characteristics, child and parent variables, and school functioning in adolescents with chronic headache: A comparison of tension-type headache and migraine. J Pediatr Psychol. 2013;38:351-364.

32. Sasmaz T, Bugdayci R, Ozge A, Karakelle A, Kurt O, Kaleagasi H. Are parents aware of their schoolchildren's headaches? Eur J Public Health. 2004;14:366-368.

33. Nakamura EF, Cui L, LateefT, Nelson KB, Merikangas KR. Parentchild agreement in the reporting of headaches in a national sample of adolescents. J Child Neurol. 2012;27:61-67.

34. Lamb ME. The history of research on father involvement: An overview. Marriage Fam Rev. 2000;29:23-42.

35. Smetana JG, Metzger A, Gettman DC, Campione-Barr N. Disclosure and secrecy in adolescent-parent relationships. Child Dev. 2006;77:201-217.

36. Børte S, Zwart JA, Stensland SØ, Hagen K, Winsvold BS. Parental migraine in relation to migraine in offspring: Family linkage analyses from the HUNT Study. Cephalalgia. 2019;39:854-862.

37. Evans S, Shipton EA, Keenan T. The relationship between maternal chronic pain and child adjustment: The role of parenting as a mediator. J Pain. 2006;7:236-243.

38. Lipton RB, Bigal ME, Kolodner K, Stewart WF, Liberman JN, Steiner TJ. The family impact of migraine: Population-based studies in the USA and UK. Cephalalgia. 2003;23:429-440.

39. Fagan MA. Exploring the relationship between maternal migraine and child functioning. Headache. 2003;43:1042-1048.

40. Leonardi M, Raggi A. A narrative review on the burden of migraine: When the burden is the impact on people's life. J Headache Pain. 2019;20:41.

41. Güngen BD, Aras YG, Gül SS, et al. The effect of maternal migraine headache on their children's quality of life. Acta Neurol Belg. 2017;117:687-694.

42. Buse DC, Powers SW, Gelfand AA, et al. Adolescent perspectives on the burden of a parent's migraine: Results from the CaMEO study. Headache. 2018;58:512-524.

43. Seng EK, Mauser ED, Marzouk M, Patel ZS, Rosen N, Buse DC. When mom has migraine: An observational study of the impact of parental migraine on adolescent children. Headache. 2019;59:224-234.

44. Arruda MA, Bigal ME. Migraine and behavior in children: Influence of maternal headache frequency. J Headache Pain. 2012;13:395-400.

45. Andersen A, Holstein BE, Berntsson L, Hansen EH. Parental symptoms and children's use of medicine for headache: Data reported by parents from five Nordic countries. Int J Public Health. 2012;57:217-223. 
46. Palermo TM, Valrie CR, Karlson CW. Family and parent influences on pediatric chronic pain: A developmental perspective. Am Psychol. 2014;69:142-152.

47. Sameroff A. A unified theory of development: A dialectic integration of nature and nurture. Child Development. 2010;81:6-22.

48. Perry NB, Dollar JM, Calkins SD, Keane SP, Shanahan L. Childhood self-regulation as a mechanism through which early overcontrolling parenting is associated with adjustment in preadolescence. Dev Psychol. 2018;54:1542.

49. Kooiman C, Spinhoven P, Trijsburg R. The assessment of alexithymia: A critical review of the literature and a psychometric study of the Toronto Alexithymia Scale-20. J Psychosomatic Res. 2002;53:1083-1090.

50. Goleman D. Emotional Intelligence. New York, NY: Bantam Books; 1995.

51. Yürümez E, Akça ÖF, Uğur Ç, Uslu RI, Kılıç BG. Mothers' alexithymia, depression and anxiety levels and their association with the quality of mother-infant relationship: A preliminary study. Int J Psychiatry Clin Pract. 2014;18:190-196.

52. Natalucci G, Faedda N, Quinzi A, et al. Alexithymia, metacognition, and theory of mind in children and preadolescents with migraine without aura (MWoA): A case-control study. Front Neurol. 2019;10:774.

53. Gatta M, Canetta E, Zordan M, et al. Alexithymia in juvenile primary headache sufferers: A pilot study. $J$ Headache Pain. 2011;12:71-80.

54. Cerutti R, Valastro C, Tarantino S, et al. Alexithymia and psychopathological symptoms in adolescent outpatients and mothers suffering from migraines: A case control study. J Headache Pain. 2016;17:39.

55. Tarantino S, De Ranieri C, Dionisi C, et al. Role of the attachment style in determining the association between headache features and psychological symptoms in migraine children and adolescents. An analytical observational case-control study. Headache. 2017;57:266-275.

56. Hexel M. Alexithymia and attachment style in relation to locus of control. Pers Individ Dif. 2003;35:1261-1270.

57. Meins E, Jayne HW, Alexandra L. Understanding alexithymia: Associations with peer attachment style and mind-mindedness. Pers Individ Dif. 2008;45:146-152.
58. Shim EJ, Park A, Park SP. The relationship between alexithymia and headache impact: The role of somatization and pain catastrophizing. Qual Life Res. 2018;27:2283-2294.

59. Sullivan MJ. The communal coping model of pain catastrophizing: Clinical and research implications. Canadian Psychol. 2012;53:32.

60. Sullivan MJL, Bishop SR, Pivik J. The pain catastrophizing scale: Development and validation. J Psychol Assess. 1995;7:524-532.

61. Simons LE, Pielech M, Cappucci S, Lebel A. Fear of pain in pediatric headache. Cephalalgia. 2015;35:36-44.

62. Sciruicchio V, Simeone M, Foschino Barbaro MG, et al. Pain catastrophizing in childhood migraine: An observational study in a tertiary headache center. Front Neurol. 2019;10:114.

63. Hermann C, Hohmeister J, Zohsel K, Ebinger F, Flor H. The assessment of pain coping and pain-related cognitions in children and adolescents: Current methods and further development. J Pain. 2007;8:802-813.

64. Vervoort T, Goubert L, Crombez G. The relationship between high catastrophizing children's facial display of pain and parental judgment of their child' pain. J Pain. 2009;142:142-148.

65. Akbarzadeh Gh, Daniali H, Javadzadeh M, Caes L, Ranjbar S, Habibi M. The relationship of parental pain catastrophizing with parents reports of children's anxiety, depression, and headache severity. Iran J Child Neurol. 2018;12:55-66.

66. Caes L, Vervoort T, Eccleston C, Goubert L. Parents who catastrophize about their children's pain prioritize attempts to control pain. Pain. 2012;153:1695-1701.

67. Martínez MP, Sánchez AI, Miró E, Lami MJ, Prados G, Morales A. Relationships between physical symptoms, emotional distress, and pain appraisal in fibromyalgia: The moderator effect of alexithymia. J Psychol. 2015;149:115-140.

68. Makino S, Jensen MP, Arimura T, et al. Alexithymia and chronic pain: The role of negative affectivity. Clin J Pain. 2013;29: 354-361.

69. Oztop DB, Tasdelen BI, Poyrazoglu HG, et al. Assessment of psychopathology and quality of life in children and adolescents with migraine. J Child Neurol. 2016;31:837-842.

70. Balottin U, Fusar Poli P, Termine C, Molteni S, Galli F. Psychopathological symptoms in child and adolescent migraine and tension-type headache: A meta-analysis. Cephalalgia. 2013;33: 112-122. 